\title{
Effects of hydroxyapatite (0001)
}

\section{$\mathrm{Ca}^{2+} / \mathrm{Mg}^{2+}$ substitution on adsorbed D-}

\author{
ribose ring puckering
}

\section{Guillem Revilla-López, ${ }^{1,2}$ Oscar Bertran, ${ }^{3}$ Jordi Casanovas, ${ }^{4}$}

\section{Pau Turon, ${ }^{5, *}$ Jordi Puiggalí, ${ }^{6,2 * *}$ and Carlos Alemán ${ }^{6,2, *}$}

${ }^{1}$ Institut für Organische Chemie, Universität Regensburg, Universitätstrasse, 31, 93040

Regensburg, Germany

${ }^{2}$ Departament d'Enginyeria Química, E. T. S. d'Enginyeria Industrial de Barcelona

Universitat Politècnica de Catalunya, Diagonal 647, 08028 Barcelona, Spain

${ }^{3}$ Departament de Física Aplicada, EEI, Universitat Politècnica de Catalunya, Av. Pla de la Massa, 8, 08700 Igualada, Spain

${ }^{4}$ Departament de Química, Escola Politècnica Superior, Universitat de Lleida, c/ Jaume

$$
\text { II } n^{o} \text { 69, Lleida E-25001, Spain }
$$

${ }^{5}$ B. Braun Surgical, S.A. Carretera de Terrasa 121, 08191 Rubí (Barcelona), Spain

${ }^{6}$ Center for Research in Nano-Engineering, Universitat Politècnica de Catalunya, Campus Sud, Edifici C', C/Pasqual i Vila s/n, Barcelona E-08028, Spain

\footnotetext{
pau.turon@bbraun.com, jordi.puiggali@upc.edu and carlos.aleman@upc.edu
} 


\begin{abstract}
Advanced Molecular Dynamics (MD) simulation protocols have been used to asses the ring puckering of cyclic D-ribose when the sugar is adsorbed on the most stable (0001) facet of calcium hydroxyapatite (HAp). In addition, sugar $\cdots$ mineral interactions, which are crucial for transfection processes and prebiotic chemistry, have been studied for systems in which the $\mathrm{Ca}^{2+}$ ions of the above mentioned HAp facet were totally or partially replaced by $\mathrm{Mg}^{2+}$. The latter replacement is spatially and quantitatively limited and has been found to cause important alterations in the conformational behavior of the D-ribose that are similar to those suffered in hairpin RNA from A to B helical structures. Accordingly, replacement of $\mathrm{Ca}^{2+}$ by $\mathrm{Mg}^{2+}$ has a dramatic effect in the functionality of the nucleic acid. These changes have been related to both the substitution site in the surface and the amount of ions. Our results show that when replacement by $\mathrm{Mg}^{2+}$ occurs in $\mathrm{OH}^{-}$-coordinated $\mathrm{Ca}^{2+}$ ions, $\mathrm{Mg}^{2+} \ldots \mathrm{D}$-ribose interactions are strong enough to prevent the interactions between the hydroxyl groups of the sugar and the remaining $\mathrm{Ca}^{2+}$ ions.
\end{abstract}




\section{INTRODUCTION}

Hydroxyapatite (HAp) is a biocompatible material that forms most of bones and teeth mass. In addition, it has been proposed to play a significant role in the origin and evolution of life $\mathrm{e}^{1,2}$ due to its ability to bind bioactive biomolecules, such as nucleic acids in the case of hydroxyolites. ${ }^{2,3}$ Biological HAp shows a different composition than that synthesized in the laboratory, $\left[\mathrm{Ca}_{10}\left(\mathrm{PO}_{4}\right)_{6}(\mathrm{OH})_{2}\right]$, since it may contain other molecules and elements. ${ }^{4}$ Among the latter ones, the presence of $\mathrm{Mg}^{2+}$ in biological HAp replacing $\mathrm{Ca}^{2+}$ plays a key role in different biological aspects of the mineral, like bone homeostasis metabolism stimulating osteogenesis ${ }^{5}$ and enhancement of material resistance to mechanical stress. ${ }^{6}$ Those effects are attributed to the sole presence of $\mathrm{Mg}^{2+}$, even though the incorporation of such ion to the mineral was predicted to be limited in the percentage of $\mathrm{Ca}^{2+}$ mass replaced ${ }^{7}$ and selective in terms of inclusion zone. ${ }^{7,8}$ More specifically, $\mathrm{Mg}^{2+}$ preferentially replaces $\mathrm{OH}^{-}$-coordinated $\mathrm{Ca}^{2+}$ atoms in the surface and sub-surface areas of the mineral. ${ }^{7,8}$

Little is known about the true influence of the position replacement by $\mathrm{Mg}^{2+}$ and the mechanism by which it modulates the interaction of the mineral with the surrounding environment. This is expected to be strongly affected by the intimate interaction between the surface of the mineral and the different biomolecules at the mineral/medium interphase. The most stable surface of HAp is the basal plane (0001) of the hexagonal cell in which the columns of hydroxide alternate between all up and all down arrangements, thus changing the symmetry group from $P 6_{3} / m$ to $P 6_{3} .{ }^{5}$ This plane has been proved to be the most active during the biomineralization process ${ }^{9}$ and it is favorable for the establishment of attractive electrostatic interactions (i.e exothermic) with charged groups of biomacromolecules like DNA ${ }^{10}$ and RNA. ${ }^{11}$ Thus, nucleic acids fit themselves into the surface pattern. The inherent flexibility of these charged 
biomacromolecules is mainly due to the torsional rotation around chemical bonds, the sugar moiety of nucleic acids playing a crucial role for this purpose.

The sugar in nucleic acids can be the cyclic D-ribose and 2-deoxy-D-ribose for RNA and DNA respectively. Furthermore, D-ribose was identified as a sugar product in several experiments reproducing the synthesis of organic components in the primitive earth environment. ${ }^{12}$ This feature enables the possibility of primitive nucleic acid synthesis from their unit components: phosphate, cyclic pentose and nitrogenous base. This synthetic process is expected that took place on an immobilizing support, like mineral HAp. Once formed, the influence of cyclic sugars on the conformational properties of nucleic acids has been widely studied highlighting the consequences of their functionalization in fields such as expression modulation ${ }^{13}$ and structural stabilization. ${ }^{14}$ Consequently, the intimate interaction between the furanose ring of the sugars and the HAp surface may have severe consequences not only for the 3D structure of nucleic acids during the adsorption ${ }^{10}$ and biomineralization ${ }^{3}$ processes, but it might be also important for understanding the initial steps towards the abiotic synthesis of nucleic acids. $^{12}$

Our previous work was focused on the embedding of $\beta$-D-ribofuranose 5-phosphate into HAp mineral. ${ }^{11}$ The latter significantly altered the conformational preferences of the ring puckering by sequestering the minimum towards ${ }^{3} \mathrm{~T}_{2}$ and reducing the conformational variability observed for standalone $\beta$-D-ribofuranose 5 -phosphate and $\beta$ D-ribofuranose itself. ${ }^{11}$ However, there is no clear view of the conformational behavior of D-ribose when it is adsorbed on the most stable facet of HAp, as there is no evidence of the conformational changes in the ring puckering and their relationship with nucleic acids structure. 
The present work assesses the conformational preferences of the cyclic D-ribose when adsorbed onto the (0001) facet of the HAp (hereafter Ca-HAp model). Furthermore, the impact of the $\mathrm{Ca}^{2+}$ substitution by $\mathrm{Mg}^{2+}$ on the puckering of the sugar ring has been examined by considering models in which the $\mathrm{Ca}^{2+}$ ions of the above mentioned Ca-HAp mineral were totally or partially replaced by $\mathrm{Mg}^{2+}(\mathrm{Mg}-\mathrm{HAp}$ or $\mathrm{Ca} / \mathrm{Mg}-\mathrm{HAp}$ model, respectively).

\section{MOLECULAR MODEL FOR THE MINERAL}

The hexagonal HAp crystal unit cell ${ }^{15}$ with $P 6_{3} / m$ geometry $(a=b=9.421 \AA, c=$ $6.881 \AA, \alpha=\beta=90^{\circ}$, and $\gamma=120^{\circ}$ ) and the $4 \mathrm{e}$ Wyckoff position occupied by two hydroxyl ions, each with $1 / 2$ occupancy, and group symmetry $P 6_{3}$ was generated. The (0001) facet was built from the latter structure by considering a $20 \times 20$ supercell reconstruction with a mineral thickness in the $z$ direction corresponding to 10 unit cells

(i.e. $>20 \AA$ ). This system, which corresponds to the Ca-HAp, model is displayed in Figure 1a. The model in which all $\mathrm{Ca}^{2+}$ of $\mathrm{Ca}-\mathrm{HAp}$ were replaced by $\mathrm{Mg}^{2+}$ ones (Figure 1b) was considered to investigate the effect of the total substitution on D-ribose puckering. Finally, the partially replaced surface, which corresponds to the $\mathrm{Ca} / \mathrm{Mg}-\mathrm{HAp}$ model (Figure 1c), was constructed by replacing the outermost $\mathrm{OH}^{-}$-coordinated $\mathrm{Ca}^{2+}$ ions by $\mathrm{Mg}^{2+}$ up to $2.5 \% \mathrm{w} / \mathrm{w} \mathrm{HAp}$, as it was reported in our previous work. ${ }^{7}$

\section{METHODS}

The conformational exploration of the initially relaxed D-ribose was carried out through by Simulated Annealing (SA) by combining classical Molecular Dynamics (MD) and conjugate gradient (CG) minimization. SA protocol was reproduced by performing $100 \mathrm{MD}-\mathrm{NVT}$ productive trajectories (i.e. MD runs used for the analyses) at 
$298 \mathrm{~K}$, of length $5 \mathrm{~ns}$ each, interleaved by a $1 \mathrm{~ns}$ MD-NVT heating up to $400 \mathrm{~K}$ and successive quenching by $\mathrm{CG}$ minimization. $\mathrm{MD}$ simulations and $\mathrm{CG}$ minimizations were performed using the NAMD software package. ${ }^{16}$ Accordingly, the total simulation time was $600 \mathrm{~ns}$ for each simulated system.

The potential energy was computed using the Amber force-field. ${ }^{17}$ Force-field parameters for D-ribose (with exception of electrostatic charges), $\mathrm{OH}^{-}$and $\mathrm{PO}_{4}^{3-}$ groups were extracted from Amber ff03. ${ }^{18}$ Electrostatic charges for D-ribose were calculated for the minimal energy structures described in our previous work ${ }^{11}$ using the Restrained Electrostatic Potential (RESP) method, ${ }^{19,20}$ and Boltzmann averaged. Force-field parameters for $\mathrm{Ca}^{2+}$ and $\mathrm{Mg}^{2+}$ were taken from Bradbrook et al. ${ }^{21}$ and Allner et al. ${ }^{22}$ respectively. It should be noted that $\mathrm{ff} 03$ force-field parameters, which are identical to the $\mathrm{ff} 99-\mathrm{SB}^{23}$ ones for nucleic acids, are able to reproduce the inorganic $\cdots$ organic interactions in biominerals, as was recently proved. ${ }^{24}$ In order to facilitate the random walk of the sugar ring over the mineral surface without causing temperature mediated desorption, artificial non-bonding parameters were assigned to the mineral atoms during the heating up runs. More specifically, these artificial parameters consisted in: $i$ ) neutral charge; and $\mathrm{ii})$ a reduction $(10 \%)$ of the van der Waals depth well $(\varepsilon)$. The mineral was kept frozen at its original crystallographic positions during the whole search process, no previous ab initio optimization being carried out. This decision was taken on the basis of the following features: 1) the (0001) is most stable surface of HAp and previous theoretical studies ${ }^{25}$ evidence a very significant agreement between ab initio and crystallographic coordinates; and 2) the conformational search has been carried out using classical MD and, therefore, the combination of force field data with experimental coordinates is apparently more reliable than with ab initio coordinates. 
In all cases, the Berendsen thermostat was used. ${ }^{26}$ The selected time step was $1 \mathrm{fs}$ and electrostatic and van der Waals non-bonding interactions were explored within a cutoff radius of $50 \AA$. Trajectory snapshots were extracted each 100 ps from the productive trajectories, thus rendering a total of 5000 structures for analysis of each mineral. According to Altona et al., ${ }^{27,28}$ the conformational preferences of D-ribose were examined considering the ring puckering amplitude, $\tau_{\mathrm{m}}$, and phase angle, $P$, of the furanose ring. Both $P$ and $\tau_{m}$ are defined by endocyclic torsion angles, as is llustrated in Figure 2a. The conformational preferences of D-ribose have been described by the pseudorotational wheel displayed in Figure 2b, in which the angular coordinate deploys the phase angle, $P$, and the concentric circles represent the puckering amplitude, $\tau_{\mathrm{m}}$. The interaction energy between the mineral and the sugar was estimated by calculating the electrostatic and van der Waals interactions between the atoms of each component (i.e. interactions between atoms belonging to the same component were omitted). In aqueous environment a continuum medium was imposed to reproduce the electrostatic effects of water following a Generalized Born model. ${ }^{29}$

\section{RESULTS AND DISCUSSION}

The role played by the conformational preferences of D-ribose in nucleic acids structure is almost entirely determined by the puckering of the furanose ring. Thus, careful inspection of ring puckering of HAp-adsorbed D-ribose is compulsory to ascertain the effects of adsorption on the sugar ring and concomitantly on the nucleic acid. Figure 3 shows the pseudorotational wheel for the three considered systems. For a given snapshot, $P$ defines the part of the ring that is most puckered while $\tau_{\mathrm{m}}$ indicates the extension of such puckering. The pseudorotation cycles depicted in Figure 3, in which $P$ varies from $0^{\circ}$ to $360^{\circ}$ through a set of 20 distinct twist and envelope (T and $\mathrm{E}$, 
respectively) arrangements, have been sub-classified into North $\left(\mathrm{N}, P=0^{\circ}\right)$, East $(\mathrm{E}, P=$ $\left.90^{\circ}\right)$, South $\left(\mathrm{S}, P=180^{\circ}\right)$ and West $\left(\mathrm{W}, P=270^{\circ}\right)$, regions. In the same figure, structures with the lower interaction energies have been marked with bigger blue squares and red triangles for the aqueous and gas-phase environments, respectively.

Inspection of Figure 3 reveals that the pseudorotational itineraries of the D-ribose are similar for the three considered minerals. More specifically, the different regions of the pseudorotational wheel are not homogeneously visited, as reflects the accumulated polar plot included in the same figure. Thus, the latter plot shows a clear preference for the South-West (SW) region of the pseudorotational cycle, which is partially coincident with the preferences of D-ribose in hairpin RNA adopting B-form helix, usually in the S region with $P$ around $190^{\circ} .{ }^{29,30}$ The $\mathrm{N}$ region, which corresponds to the A-form of $\mathrm{RNA}^{29,30}$ is also present in the accumulated plot but much less populated than the SW one. The SW-to-N shift in the puckering preferences of RNA is frequently explained by the formation of specific interactions between the hydroxyl attached to the $\mathrm{C} 2$ ' atom of the sugar and nitrogen atoms of the nitrogenous base in other nucleotides. Although these interactions are not possible when D-ribose is considered alone, the electrostatic pattern at the minerals surface, which depends on the position of the different ions, provides a surrogate mold to reproduce a similar effect.

The interaction energy between the sugar and the three different minerals has been calculated by adding the cross energy components associated to the electrostatic and van der Waals interactions. Those structures within a relative interaction energy below 10 $\mathrm{kcal} / \mathrm{mol}$ in the gas phase are represented in Figure 3 as red triangles, whereas those fulfilling the same criteria when an implicit aqueous environment is applied are depicted as blue squares. In both cases the size of the symbol increases with decreasing relative interaction energy. It should be emphasized that the represented structures correspond to 
the interaction modes that are mostly favored and, therefore, provide information about how the mineral interface might alter the conformational preferences of the sugar ring and by extension of whole nucleic acid.

Inspection of the results obtained in the gas phase reveals that the substitution of $\mathrm{Ca}^{2+}$ by $\mathrm{Mg}^{2+}$ into the HAp surface to produce $\mathrm{Ca} / \mathrm{Mg}$-HAp alters the interaction mode of D-ribose with the mineral. Thus, although the more favorable interaction patterns are observed around the North-West (NW) and $\mathrm{W}$ regions for both $\mathrm{Ca}-\mathrm{HAp}$ and $\mathrm{Ca} / \mathrm{Mg}-$ HAp, the conformational flexibility (i.e. the number of minima below a $10 \mathrm{kcal} / \mathrm{mol}$ threshold) is significantly higher for the latter than for the former (i.e. 3 and 9 minima for Ca-HAp and $\mathrm{Ca} / \mathrm{Mg}-\mathrm{HAp}$, respectively). Thus, the partial substitution by $\mathrm{Mg}^{2+}$ strongly reduces the accessibility of the most likely interaction mode, as it is evidenced by the fact that the gap between the two lowest energies widens from 5 to $8 \mathrm{kcal} / \mathrm{mol}$. This can only be attributed to the combined effect of $\mathrm{Ca}^{2+}$ and $\mathrm{Mg}^{2+}$. This feature is corroborated by the fact that the only thermodynamically available interaction mode in the hypothetical Mg-HAp mineral is located at the SE region, where only $\mathrm{Ca} / \mathrm{Mg}-\mathrm{HAp}$ shows an accessible minimum.

Comparison of the lowest energy interaction modes in the gas phase indicates an important difference, which entails a shift in the preferred sugar puckering. More specifically, the O-exo $\left(\mathrm{E}_{\mathrm{O}}\right)$ is the most favored conformation for D-ribose adsorbed onto Ca-HAp while the $\mathrm{C} 1$-endo $\left({ }^{1} \mathrm{E}\right)$ is the preferred onto $\mathrm{Ca} / \mathrm{Mg}-\mathrm{HAp}$, as can be seen in Figure 3 (red triangles). This change indicates that the partial replacement of $\mathrm{Ca}^{2+}$ by $\mathrm{Mg}^{2+}$ mostly reverse the RNA B-form tendency found for Ca-HAp. In contrast, the global minimum for Mg-HAp corresponds to the $\mathrm{C} 1$-exo-O-endo $\left({ }^{\mathrm{O}} \mathrm{T}_{1}\right)$ conformation, which has no relation with RNA B-for or A-form. 
In general, results obtained using the implicit aqueous environment are very similar in terms of accumulation plots to those displayed in Figure 3 and, therefore, have not been represented. However, due to changes in the stability of the most favored modes, especially for $\mathrm{Mg}-\mathrm{HAp}$ and $\mathrm{Ca} / \mathrm{Mg}-\mathrm{HAp}$, aqueous solution structures with relative interaction energies lower than $10 \mathrm{kcal} / \mathrm{mol}$ (blue squares) have been included in Figure 3. As it can be seen, the most stable puckering obtained onto Ca-HAp is at the $\mathrm{W}$ region for both gas-phase and aqueous environment $\left(P / \tau_{\mathrm{m}}=280^{\circ} / 54^{\circ}\right.$ and $268^{\circ} / 35^{\circ}$, respectively). In contrast, the lowest interaction mode onto Mg-HAp changes from the E region in the gas-phase $\left(P / \tau_{\mathrm{m}}=106^{\circ} / 53^{\circ}\right)$ to the opposite $\mathrm{W}$ region in aqueous solution $\left(P / \tau_{\mathrm{m}}=261^{\circ} / 38^{\circ}\right)$. Changes are even more important onto the $\mathrm{Ca} / \mathrm{Mg}-\mathrm{HAp}$ mineral: the lowest interaction changes from the NW region $\left(P / \tau_{\mathrm{m}}=310^{\circ} / 43^{\circ}\right)$ in the gas-phase to the SE one in aqueous solution $\left(P / \tau_{\mathrm{m}}=129^{\circ} / 31^{\circ}\right)$. In addition, the number of favorable interaction modes significantly decreases in the latter environment.

In a recent study we calculated the pseudorotational puckering of $\beta$-D-ribofuranose and $\beta$-D-ribofuranose 5-phosphate in the gas-phase and of the latter anchored to a CaHAp mineral using Density Functional Theory (DFT) calculations. ${ }^{11}$ The lowest energy puckering of $\beta$-D-ribofuranose in the gas-phase corresponded to the $\mathrm{C} 2$-exo-C3-endo $\left({ }^{3} \mathrm{~T}_{2}\right.$ at $P=0^{\circ}, \mathrm{N}$ region) while the $\mathrm{C} 1$-exo-C2-endo $\left({ }^{2} \mathrm{~T}_{1}\right.$ at $P=140^{\circ}, \mathrm{SE}$ region $)$ and the C4-endo $\left({ }^{4} \mathrm{E}\right.$ at $P=234^{\circ}, \mathrm{SW}$ region $)$ were identified as secondary minima. The stability of such three minima $\left({ }^{3} \mathrm{~T}_{2},{ }^{2} \mathrm{~T}_{1}\right.$ and $\left.{ }^{4} \mathrm{E}\right)$ was found to be approximately the same for $\beta$-Dribofuranose 5-phosphate in the gas-phase. ${ }^{11}$ It is worth noting that results presented in Figure 3 indicates that, the SW region is the most abundant when the sugar is adsorbed onto a HAp (0001) facet, independently of the $\mathrm{Ca}^{2+}$ by $\mathrm{Mg}^{2+}$ substitution. Accordingly, our current MD simulations reflect a selective enrichment of the minima detected by DFT calculations. 
The most sounding comparison is between the puckering predicted in this work for the adsorbed D-ribose onto Ca-HAp and the minima calculated for $\beta$-D-ribofuranose 5phosphate embedded into Ca-HAp. For the latter case, the global minimum was identified at $P=0^{\circ}$, the same that for the free standing sugar, while a secondary higher energy structure was found at $P=180^{\circ} .{ }^{11}$ Comparison of those results with the accumulated data of our simulations (Figure 3) makes clear that the puckering minima in the adsorbed D-ribose resembles more those obtained for $\beta$-D-ribofuranose and $\beta$-Dribofuranose 5-phosphate rings in the gas-phase than the ones for latter compound embedded into Ca-HAp. Thus, formation of a covalent bond between a phosphate group of the mineral and the $\mathrm{C}^{\prime}$ '-OH strongly distorts the conformational preferences of the sugar not only by changing the global minimum but also the availability of the remaining.

In summary, the puckering of the adsorbed D-ribose experiences a shift with respect to the free molecule. The preferred conformations change towards arrangements that are less representative for the free-standing molecule, while the representativeness of the global minima for the free ring is reduced. Moreover, there are significant differences between the HAp embedded sugar, with only one significant minimum at $P=0^{\circ}$, and the adsorbed state on Ca-HAp and $\mathrm{Ca} / \mathrm{Mg}-\mathrm{HAp}$ discussed above, which clearly entail a progressive loss of flexibility as mineral nucleates around the sugar.

Figure 4 shows the probability distribution for atom pair correlation distances below $5 \AA$. The chosen pairs include all atoms where both electrostatic and van de Waals energy contributions play a relevant role. These include: hydrogen bonds between the $\mathrm{OH}$ donor groups of the D-ribose and oxygen phosphate atoms, polar-charged interactions between oxygen atoms in the sugar and $\mathrm{Ca}^{2+}$ or $\mathrm{Mg}^{2+}$, and hydrogen bonds between the $\mathrm{OH}$ groups of the sugar and the $\mathrm{OH}^{-}$of the mineral. These distributions 
offer a sight of the dominating interactions between the biomolecule and the mineral slab, shedding light on the mechanism of how the differential energy contributions dominate alterations in the D-ribose puckering.

Comparison of the probability functions calculated for $\mathrm{Ca}-\mathrm{HAp}$ and $\mathrm{Ca} / \mathrm{Mg}-\mathrm{HAp}$ reveals that the partial substitution of $\mathrm{Ca}^{2+}$ by $\mathrm{Mg}^{2+}$ in the surface leads to an almost complete loss of the interactions between the $\mathrm{Ca}^{2+}$ and the oxygen atoms of the $\mathrm{D}$ ribose. Thus, although such interactions are predominant in Ca-HAp (Figure 4a), no relevant peak appears up to $3.5 \AA$ in $\mathrm{Ca} / \mathrm{Mg}$-HAp (Figure $4 \mathrm{~b}$ ). This change is logically accompanied by the apparition of interactions between $\mathrm{Mg}^{2+}$ and the oxygen atoms of D-ribose. The interaction pattern of $\mathrm{Mg}^{2+} \cdots \mathrm{D}$-ribose is fairly similar to that of $\mathrm{Ca}^{2+} \cdots \mathrm{D}-$ ribose. Thus, the first peak for $\mathrm{Mg}^{2+} \cdots \mathrm{D}$-ribose soars at $\sim 0.5 \AA$, while several peaks appear around $2.0 \AA$ for $\mathrm{Ca}^{2+} \ldots \mathrm{D}$-ribose. This has been attributed to the fact that $\mathrm{Ca}^{2+}$ replacement by $\mathrm{Mg}^{2+}$ happens in $\mathrm{OH}^{-}$-coordinated cations, whereas those $\mathrm{PO}_{4}^{3-}$ coordinated remain unchanged. This result suggests that $\mathrm{Mg}^{2+} \cdots \mathrm{D}$-ribose interactions are stronger enough to prevent the interaction of oxygen atoms of D-ribose with the remaining $\mathrm{Ca}^{2+}$ ions in HAp.

In the Mg-HAp system the peaks are preserved below $2.0 \AA$ compared the $\mathrm{Ca} / \mathrm{Mg}$ HAp system, reflecting interactions between the oxygen atoms of D-ribose and the $\mathrm{Mg}^{2+}$ ions (Figures $4 \mathrm{~b}$ and $4 \mathrm{c}$ ). This observation reinforces the idea that $\mathrm{OH}^{-}$-coordinated $\mathrm{Mg}^{2+}$ "sequesters" the sugar, whereas when $\mathrm{PO}_{4}^{3-}$-coordinated $\mathrm{Ca}^{2+}$ are also replaced by $\mathrm{Mg}^{2+}$, the interaction with these sites appears again.

Inspection of the interactions between the $\mathrm{HO}$ moieties of D-ribose and the oxygen atoms of the $\mathrm{PO}_{4}^{3-}$ groups reveals a fairly similar pattern in Ca-HAp and $\mathrm{Ca} / \mathrm{Mg}-\mathrm{HAp}$, though less preserved in Mg-HAp. These interactions are associated with the flexibility of the proton orientation in the OH-ribose groups. Thus, if the leading interaction 
involves the oxygen atoms of D-ribose and the cations (i.e. $\mathrm{Mg}^{2+}$ and/or $\mathrm{Ca}^{2+}$ ), the flexibility of the $\mathrm{C}-\mathrm{O}$ bonds in the sugar facilitate the hydrogen to form alternate interactions with the different oxygens of $\mathrm{PO}_{4}^{3-}$. Finally, the interaction between the $\mathrm{OH}$ groups of D-ribose and the $\mathrm{OH}^{-}$of the mineral is the less important for all three HAp systems, peaks appearing always over $3 \AA$.

The main conclusion from the inspection of the probability distributions of pair distances displayed in Figure 4 is that the replacement of $\mathrm{OH}^{-}$-coordinated $\mathrm{Ca}^{2+}$ by $\mathrm{Mg}^{2}$ strongly affects the ability of D-ribose to interact with $\mathrm{PO}_{4}^{3-}$-coordinated $\mathrm{Ca}^{2+}$, as is reflected by the changes in the corresponding peaks. However, the total replacement of $\mathrm{Ca}^{2+}$ by $\mathrm{Mg}^{2+}$ does not reverse the pattern to that of Ca-HAp. This observation proves that the changes observed in $\mathrm{Ca} / \mathrm{Mg}-\mathrm{HAp}$ are composed of both differential interaction strength and the patterning effects of the cation substitution.

Figure 5 depicts axial and longitudinal views of the HAp adsorbed D-ribose in the Ca-HAp, Mg-HAp and Ca/Mg-HAp systems. These pictures provide an idea of how the sugar adapts to fit the different surface patterns induced by the cation exchange. As it can be seen, D-ribose is mostly placed over a column of hydroxide with the hydrogen aiming upwards and downwards for $\mathrm{Ca}-\mathrm{HAp}$ and $\mathrm{Ca} / \mathrm{Mg}-\mathrm{HAp}$, respectively. Such location results in the formation of close interactions between the oxygen atoms of Dribose and the $\mathrm{Ca}^{2+}$ and $\mathrm{PO}_{4}^{3-}$ ions of $\mathrm{Ca}-\mathrm{HAp}$ and $\mathrm{Ca} / \mathrm{Mg}-\mathrm{HAp}$, respectively. These dispositions contrast with that observed for Mg-HAp, in which the D-ribose interacts more closely with the $\mathrm{Mg}^{2+}$ and, consequently, shifts away from the hydroxide column center. Arrangements displayed in Figure 5 are in complete agreement with the interactions extracted from the probability distribution functions discussed above. Thus, Figures $4 \mathrm{a}$ and $4 \mathrm{c}$ reflect similar interaction peaks below $2.5 \AA$ for $\mathrm{Ca}^{2+}$ and $\mathrm{Mg}^{2+}$ when interacting with the sugar oxygen atoms in the Ca-HAp and the Mg-HAp systems, 
respectively, whilst the D-ribose $\cdots \mathrm{PO}_{4}^{3-}$ are the sole representative interaction in this range for $\mathrm{Ca} / \mathrm{Mg}-\mathrm{HAp}$ (Figure $4 \mathrm{~b}$ ).

\section{CONCLUSIONS}

The conformational preferences of D-ribose adsorbed onto the (0001) facet of HAp have been assessed by employing an indicator like the ring pseudorotation puckering, which serves to trace conformational changes on bigger molecules like RNA. Besides, the effect of $\mathrm{Ca}^{2+}$ substitution by $\mathrm{Mg}^{2+}$, which is limited in quantity to a maximum of $2.5 \%(\mathrm{w} / \mathrm{w})$ and in space distribution to the surface and subsurface areas, has been assessed by employing two different surface substitution patterns. The $\mathrm{Ca}^{2+}$ by $\mathrm{Mg}^{2+}$ substitution alters the pseudorotational preferences of the sugar in the regions related to the A to B helix structure transition in longer RNA chains, indicating that this phenomenon can be mediated by the surface. Divergences in the conformational preferences are explained by the different affinity of $\mathrm{Mg}^{2+}$ and $\mathrm{Ca}^{2+}$ ions towards the different interacting groups of the sugar which, ultimately, changes the preferred adsorption sites and the induced fitting of the ring. Interestingly, replacement of $\mathrm{OH}^{-}$coordinated $\mathrm{Ca}^{2+}$ by $\mathrm{Mg}^{2}$ strongly affects $\mathrm{Ca}^{2+} \cdots \mathrm{D}$-ribose interactions. Thus, $\mathrm{Mg}^{2+} \cdots \mathrm{D}-$ ribose interactions in $\mathrm{Ca} / \mathrm{Mg}-\mathrm{HAp}$ inhibit the interactions of D-ribose with the $\mathrm{PO}_{4}^{3-}$ coordinated $\mathrm{Ca}^{2+}$ ions.

\section{ACKNOWLEDGEMENTS}

Authors acknowledge MINECO-FEDER (MAT2015-69367-R and MAT201569547-R) for financial support. Support for the research of C.A. was received through the prize "ICREA Academia" for excellence in research funded by the Generalitat de 
Catalunya. This work is integrated within a wider research project supported by B. Braun Surgical S.A., UPC and other institutions.

\section{REFERENCES}

1. S. M. Porter, Science, 2007, 316, 1302-1304.

2. P. Turon, J. Puiggalí, O. Bertran and C. Alemán, Chem. Eur. J., 2015, 21, $18892-18898$

3. O. Bertran, L. J. del Valle, G. Revilla-López, G. Chaves, L. Cardus, M. T. Casas, J. Casanovas, P. Turon, J. Puiggalí and C. Alemán, Dalton Trans., 2014, 43, $317-327$.

4. J. C. Elliott, Structure and chemistry of the apatites and other calcium orthophosphates. In: Studies in Inorganic Chemistry, vol. 18. Amsterdam: Elsevier, 1994.

5. E. Landi, A. Tampieri, M. Mattioli-Belmonte, G. Celotti, M. Sandri, A. Gigante, P. Fava and G. Biagini, J. Eur. Ceram. Soc., 2006, 26, 2593-2601.

6. R. K. Rude and H. E. Gruber, J. Nutr. Biochem., 2004, 15, 710-716.

7. O. Bertran, L. J. del Valle, G. Revilla-López, M. Rivas, G. Chaves, M. T. Casas, J. Casanovas, P. Turon, J. Puiggalí and C. Alemán, Chem. Eur. J., 2015, 21, 2537-2546.

8. D. Laurencin, N. A. Barrios, N. H. de Leeuw, C. Gervais, C. Bonhomme, C. F. Mauri, W. Chrzanowski, J. C. Knowles, R. J. Newport, A. Wong, Z. H. Gan and M. E. Smith, Biomaterials, 2011, 32, 1826-1837.

9. A. Rimola, M. Corno, C. M. Zicovich-Wilson and P. Ugliengo, J. Am. Chem. Soc., 2008, 130, 16181-16183. 
10. L. J. del Valle, O. Bertran, G. Chaves, G. Revilla-López, M. Rivas, M. T. Casas, J. Casanovas, P. Turon, J. Puiggalí and C. Alemán, J. Mater. Chem. B, 2014, 2, 69536966.

11. J. Casanovas, G. Revilla-López, O. Bertran, L. J. del Valle, P. Turón, J. Puiggalí and C. Alemán, J. Phys. Chem. B, 2014, 118, 5075-5081.

12. R. Shapiro, Orig Life Evol Biosph., 1988, 18, 71-85.

13. N. Martín-Pintado, M. Yahyaee-Anzahaee, G. F. Deleavey, G. Portella, M. Orozco, M. J. Damha and C. González, J. Am. Chem. Soc., 2013, 135, 5344-5347.

14. N. Martín-Pintado, G. F. Deleavey, G. Portella, R. Campos-Olivas, M. Orozco, M. J. Damha and C. González, Angew. Chem. Int. Ed., 2013, 125, 12287-12290.

15. L. Stork, P. Muller, R. Dronskowski and J. R. Z. Ortlepp, Kristallogr., 2005, 220, 201-205.

16. J. C. Phillips, R. Braun, W. Wang, J. Gumbart, E. Tajkhorshid, E. Villa, C. Chipot, R. D. Skeel, L. Kale and K. Schulten, J. Comput. Chem., 2005, 26, 1781-1802.

17. W. D. Cornell, P. Cieplak, C. I. Bayly, I. R. Gould, K. M. Merz, D. M. Ferguson, D. C. Spellmeyer, T. Fox, J. W. Caldwell and P. A. Kollman, J. Am. Chem. Soc., 1995, 117, 5179-5197.

18. Y. Duan, S. Chowdhury, M. C. Lee, G. Xiong, W. Zhang, R. Yang, P. Cieplak, R. Luo, T. Lee, J. Caldwell, J. Wang and P. A. Kollman, J. Comput. Chem., 2003, 24, 1999-2012.

19. C. I. Bayly, P. Cieplak, W. D. Cornell and P. A. Kollman, J. Phys. Chem., 1993, 97, 10269-10280.

20. P. Cieplak, C. I. Bayly, W. D. Cornell and P. A. Kollman, J. Comput. Chem., 1995, 16, 1357-1377. 
21. G. M. Bradbrook, T. Gleichmann, S. J. Harrop, J. Habash, J. Raftery, J. Kalb, J. Yariv, I. H. Hillier and J. R. Helliwell, J. Chem. Soc. Faraday Trans., 1998, 94, 16031611.

22. O. Allnér, L. Nilsson and A. Villa, J. Chem. Theory Comput., 2012, 8, 14931502.

23. V. Hornak, R. Abel, A. Okur, B. Strockbine, A. Roitberg and C. Simmerling, Proteins, 2006, 65, 712-725.

24. G. Revilla-López, J. Casanovas, O. Bertran, P. Turón, J. Puiggalí and C. Alemán, Biointerphases, 2013, 8, 10.

25. M. Cormo, A. Rimola, V. Bolis and P. Ugliengo, Phys.Chem.Chem.Phys., 2010, 12, 6309 .

26. H. J. C. Berendsen, J. P. M. Postma, W. F. van Gunsteren, A. DiNola and J. R. Haak, J. Chem. Phys., 1984, 81, 3684-3690.

27. C. Altona and M. Sundaralingam, J. Am. Chem. Soc., 1972, 94, 8205-8212.

28. C. Altona and M. Sundaralingam, J. Am. Chem. Soc., 1973, 95, 2333-2344.

29. W. C. Still, A. Tempczyk, R. C. Hawley and T. Hendrickson, J. Am. Chem. Soc., 1990, 112, 6127-6129.

30. A. Palladino and R. Zangi, Biophys. Chem., 2013, 180-181, 110-118.

31. A. Sedova and N. K. Banavali, Biopolymers, 2015, 105, 65-82. 


\section{CAPTIONS TO FIGURES}

Figure 1. Axial view of the three different mineral slabs representing the (0001) facet of HAp: A) Ca-HAp; B) Mg-HAp; and C) $\mathrm{Ca} / \mathrm{Mg}-\mathrm{HAp}$, where $\mathrm{Mg}^{2+}$ (green spheres) are in $\mathrm{OH}^{-}$ coordinated positions whereas $\mathrm{Ca}^{2+}$ (blue spheres) are in $\mathrm{PO}_{4}^{3-}$ coordinated positions. The remaining color codes correspond to oxygen (red spheres) and phosphorous (orange spheres). A $2 \times 2$ supercell is marked by dark lines to show the alternate distribution of $\mathrm{OH}^{-}$columns.

Figure 2. (a) Endocyclic dihedral angles of D-ribose and expressions used to obtain the phase of pseudorotation $(P)$ and the puckering amplitude $\left(\tau_{m}\right)$. (b) Pseudorotational wheel of D-ribose. The North, East, South and West region are defined.

Figure 3. Accumulation plot for the ring puckering of D-ribose deploying the phase angle, $P$ from 0 to $360^{\circ}$, in the circular coordinate and the amplitude, $\tau_{\mathrm{m}}$ from $0^{\circ}$ to $60^{\circ}$. in the inner/outer circles for Ca-HAp, Mg-HAp and Ca/Mg-HAp. The accumulated percentages for each region of the pseudorotation are represented in the last map using the average value of each region to assign the whole representative percentage following the same previous color code: cyan for Ca-HAp; green for Mg-HAp and wine red for $\mathrm{Ca} / \mathrm{Mg}$-HAp. Red triangles represent the structures with sugar $\cdots$ mineral interaction energies below $10 \mathrm{kcal} / \mathrm{mol}$ with regard to the minimum in the gas phase, whereas blue squares take into account the implicit water correction below the same threshold. Symbol sizes reveal the energetic order, the biggest ones being the lowest energy, more likely, modes.

Figure 4. Probability distributions for pair distances below $5 \AA$ of all relevant interactions between D-ribose and (a) Ca-HAp, (b) Ca/Mg-HAp and (c) Mg-HAp. Labels follow the code: $\mathbf{h}$ for hydrogen atoms in the $\mathrm{OH}$ groups of D-ribose; hi for hydrogen atoms of the $\mathrm{OH}^{-}$groups of HAp; ox for oxygen atoms of the D-ribose; op for 
oxygen atoms of the phosphate groups of HAp; ca for $\mathrm{Ca}^{2+}$ of $\mathrm{HAp}$ and $\mathbf{m g}$ for $\mathrm{Mg}^{2+}$ of HAp.

Figure 5. Axial (upper image) and longitudinal (lower image) of the lowest D-ribose interaction energy structures on (A) Ca-HAp, A, (B) Mg-HAp and (C) Ca/Mg-HAp. Representation code is as follow: blue for $\mathrm{Ca}^{2+}$, green for $\mathrm{Mg}^{2+}$, red for oxygen, orange for phosphorous CPK color code for D-ribose. 
A $10.0405 \% 25 \%$

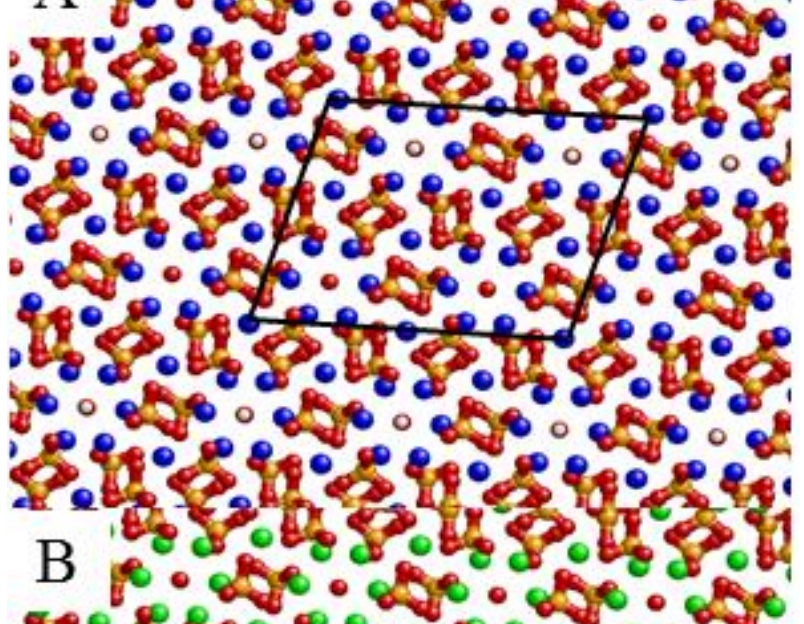

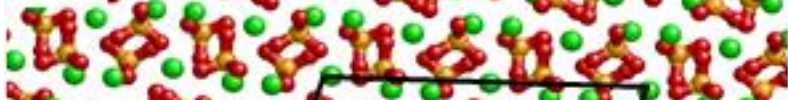

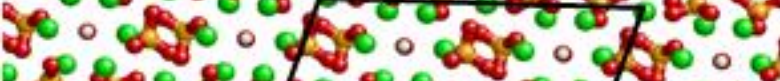
28008008008000

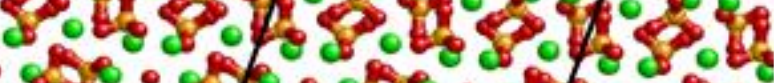

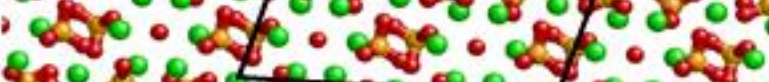

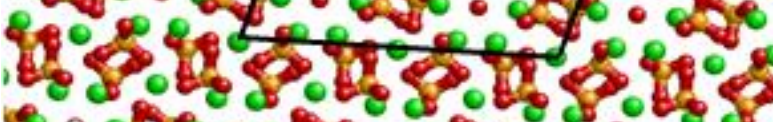
0.86 .040 480.280 .8

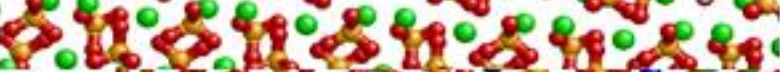
$C$ D.

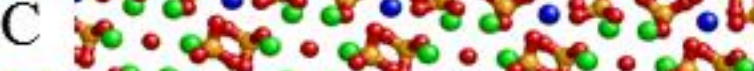

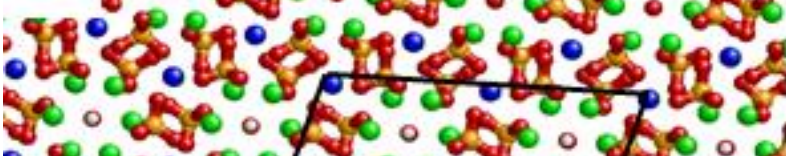

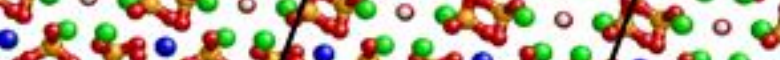

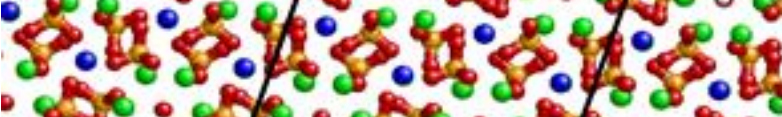

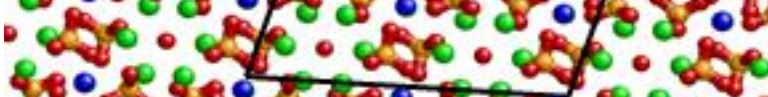
$10 \%$ कर

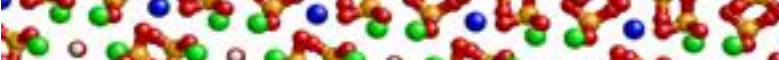

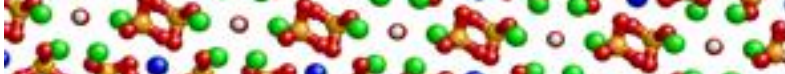
Pot

Figure 1 
(a)

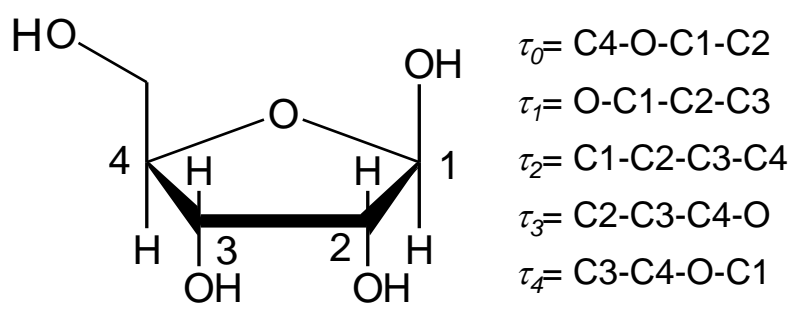

$$
\begin{aligned}
& \mathrm{P}=\tan ^{-1}\left[\frac{\left(\tau_{1}+\tau_{4}\right)-\left(\tau_{0}+\tau_{3}\right)}{3.077683 \cdot \mathrm{T}_{2}}\right] \\
& \tau_{\mathrm{m}}=\frac{\tau_{2}}{\cos \mathrm{P}}
\end{aligned}
$$

(b)

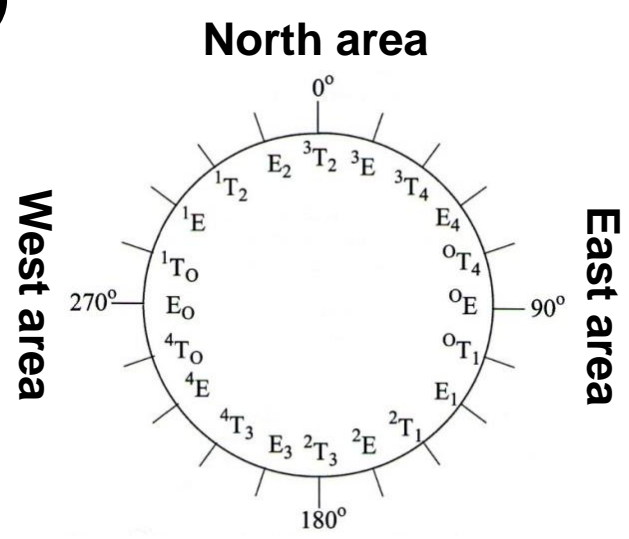

South area

Figure 2 

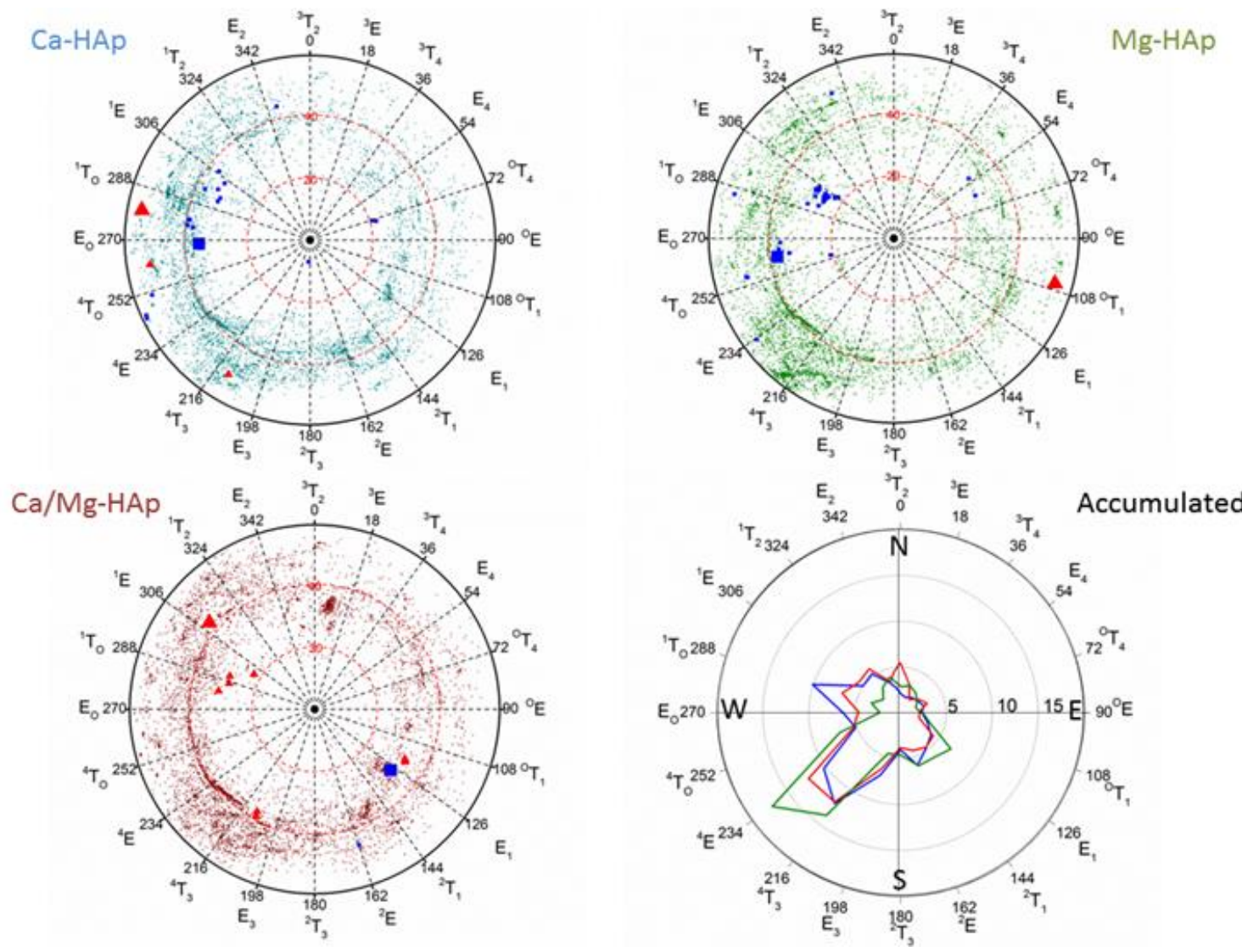

Figure 3 


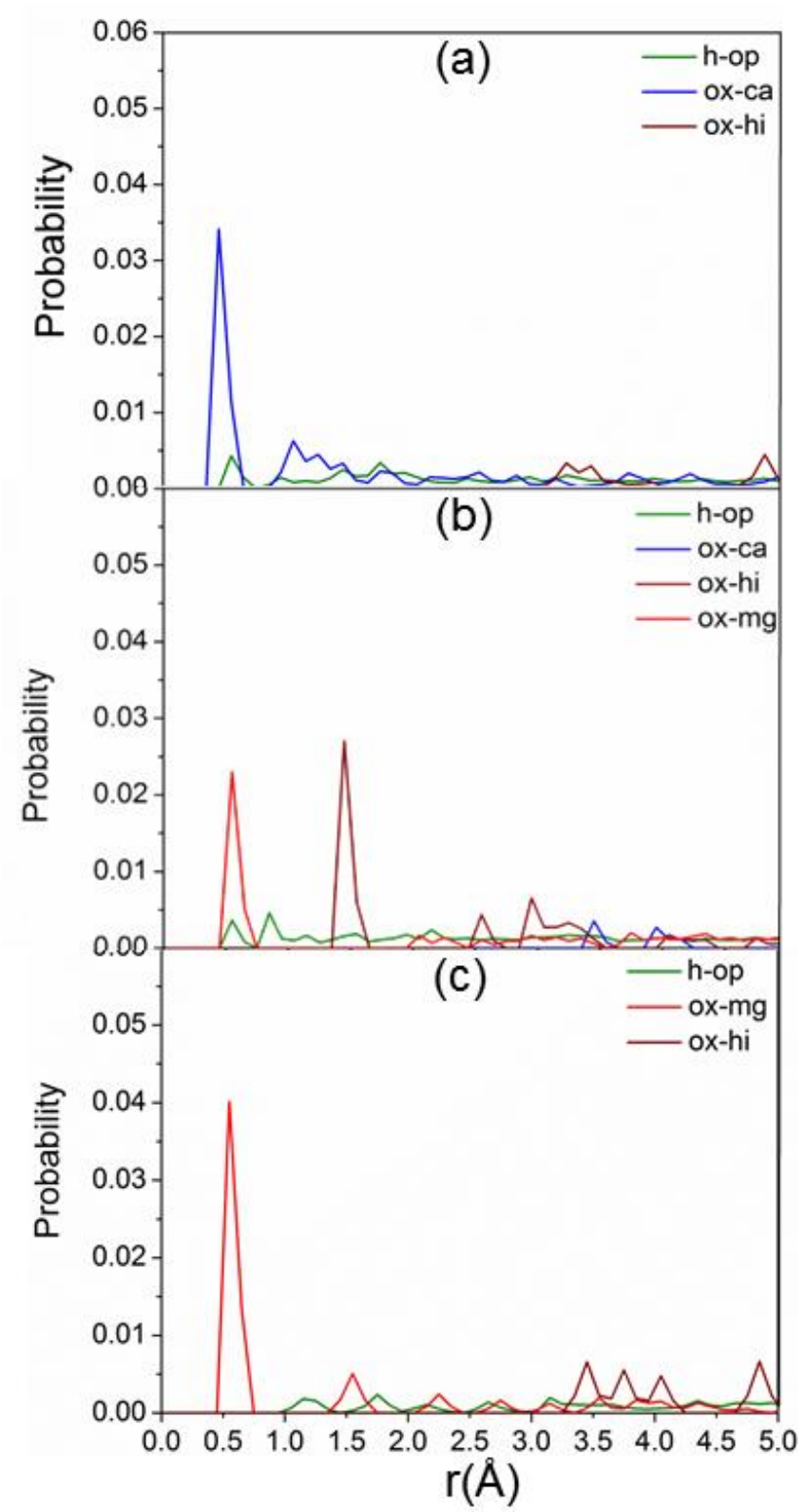

Figure 4 


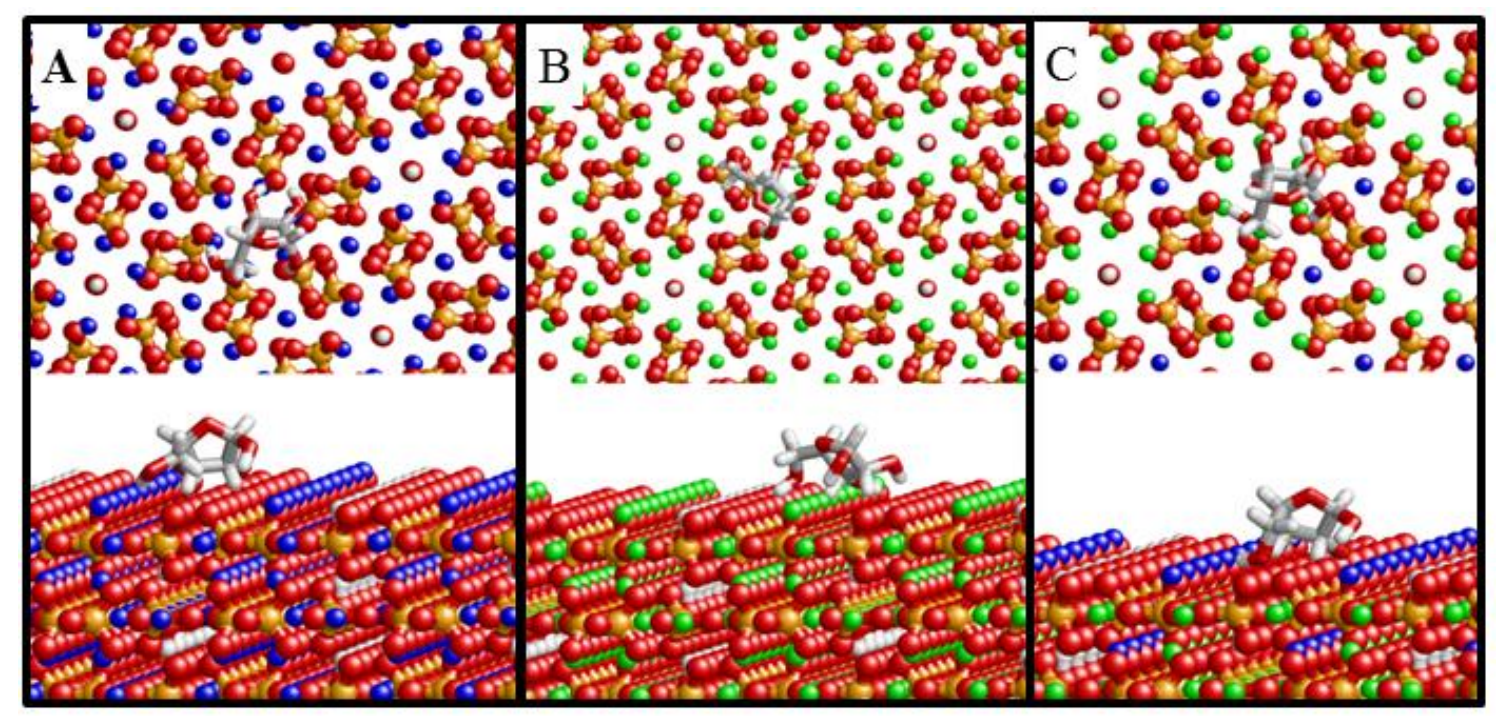

Figure 5 